\title{
Cálculo de las constantes cinéticas del proceso de termo inactivación disociativa de la fosfatasa Acida
}

\section{Quijano Parra Alfonso', Martínez Ch Oscar Luis' ${ }^{1}$,Arbeláez R Luis Fernando²}

1'Departamento de Química. Facultad de Ciencias Básicas. Grupo de Investigación en Química. Universidad de Pamplona.

${ }^{2}$ Departamento de Medicina. Facultad de Salud. Grupo de Investigación en Química. Universidad de Pamplona

\section{Resumen}

Introducción: La fosfatasa acida es una enzima oligomérica compuesta por dos subunidades y la cinética de termo inactivación de las enzimas diméricas, se realiza en dos etapas y corresponde al mecanismo cinético secuencial de termo inactivación disociativa de un dímero activo, que se disocia reversiblemente en subunidades inactivas.

Objetivo: Estudiar el proceso de termo inactivación de la fosfatasa acida a diferentes temperaturas y un pH óptimo de la enzima

Materiales y métodos: Se utilizó un preparado de fosfatasa acida del germen de trigo de la firma Sigma, USA. El sustrato utilizado es el p-nitrofenilfosfato de sodio hexahidratado de la firma Alfa Aesar A Jhonson Mathey Company. La actividad de la fosfatasa acida se determinó por la velocidad inicial de la hidrólisis del pnitrofenilfosfato de sodio. La cantidad de p-nitrofenol formado se determinó en el espectrofotómetro Genesys 20 a $405 \mathrm{~nm}$. La termo inactivación se llevó a cabo en el buffer de acetato $0.1 \mathrm{M}$ a un $\mathrm{pH}$ de $5,5 \mathrm{y}$ valores de temperatura $50^{\circ} \mathrm{C}, 55^{\circ} \mathrm{C}, 60^{\circ} \mathrm{C}$ y $65^{\circ} \mathrm{C}$

Resultados: Se estudió el esquema cinético de termo inactivación de la enzima dimérica -fosfatasa acida que incluye la disociación reversible del dímero activo en monómeros inactivos y la posterior denaturación de los monómeros. Se calculan los parámetros cinéticos característicos de la termo inactivación de una enzima dimérica $k_{1}, k_{-1}, k_{\mathrm{d}}, K_{\text {dis }}$ y $k_{\text {aso }}$ 


\section{Conclusiones:}

1.-Las curvas cinéticas en coordenadas semilogarítmicas de una ecuación de primer orden tienen un punto de quiebre.

2.- Los valores de $\mathrm{k}_{1}$ correspondientes a la constante de velocidad de disociación de la fosfatasa ácida que determinan la estructura del dímero, aumentan con la temperatura.

3.-La energía de activación de la disociación del dímero a monómero de la fosfatasa acida es de $23,22 \mathrm{KJ} / \mathrm{Mol}$

Palabras clave: Fosfatasa acida, denaturación, estructura dimérica, monómero inactivo, cinética, termo inactivación

\section{Calculating the kinetic constants of inactivation process thermo dissociative acid phosphatase}

\section{Abstract}

Introduction: The acid phosphatase is an oligomeric enzyme composed of two subunits and kinetics of thermal inactivation of the dimeric enzyme, is performed in two stages and corresponds to the sequential kinetic mechanism of thermal inactivation of a dissociative active dimer that reversibly dissociates into subunits inactive.

Objective: To study the process of heat inactivation of the acid phosphatase at different temperatures and $\mathrm{pH}$ optimum of the enzyme

Materials and methods: a preparation of acid phosphatase from wheat germ from Sigma, USA was used. The substrate used is $p$-nitrophenyl phosphate hexahydrate signature sodium Alfa Aesar Johnson Mathey A Company. The acid phosphatase activity was determined by the initial rate of hydrolysis of $p$-nitrophenyl phosphate sodium. The amount of $p$-nitrophenol formed was determined on the spectrophotometer Genesys 20-405 nm. The thermal inactivation was carried out in $0.1 \mathrm{M}$ acetate buffer at $\mathrm{pH} 5.5$ and temperature values $50{ }^{\circ} \mathrm{C}, 55^{\circ} \mathrm{C}, 60^{\circ} \mathrm{C}$ and $65^{\circ} \mathrm{C}$ Results: thermo kinetic scheme dimeric enzyme inactivation -fosfatasa acid including dimer active reversible dissociation into inactive monomers and subsequent 
denaturation of the monomers was studied. Kinetic parameters characteristic of the thermal inactivation of a dimeric enzyme were calculated k1, K-1, Kd, Kaso y Kdis.

\section{Conclusions:}

1.-The kinetic curves in semilogarithmic coordinates of a first-order equation have a breaking point.

2. The values of $k 1$ corresponding to the dissociation rate constant of acid phosphatase dimer determine the structure increase with temperature.

3.-The activation energy of dissociation of the dimer to monomer is acid phosphatase of $23.22 \mathrm{~kJ} / \mathrm{mol}$

Keywords: acid phosphatase, denaturation, dimeric structure, inactive monomer, kinetic, thermal inactivation.

*Para citar este artículo: Quijano Parra A,Martínez Ch OL.,Arbeláez R LF.Cálculo de las constantes cinéticas del proceso de termo inactivación disociativa de la fosfatasa Acida.Revista Bistua.2015.13(1):16-26

+ Autor para el envió de correspondencia y la solicitud de las separatas: Alfonso Quijano Parra.Laboratorio de Control de Calidad.Grupo de Investigación en Química. Universidad de Pamplona.. email: alfonsoquijanoparra@unipamplona.edu.co. 


\section{Introducción}

Las Fosfatasas (E.C 3.1.3) son enzimas presentes en fluidos corporales, tejidos de mamíferos, microorganismos y las plantas $^{1,2}$. Las Fosfatasas se dividen en dos grupos: las fosfatasas alcalinas (E.C.3.1.3.1) y las fosfatasas ácidas (E.C.3.1.3.2). Las fosfatasas ácidas participan en las reacciones de transfosforilización $^{3}$ y tienen una gran aplicación ambiental, ya que pueden reducir la carga de fosfato del medio ambiente ${ }^{4}$, es utilizada como indicador de pasteurización en productos cárnicos $^{5-7}$, alimentos marinos ${ }^{8}$ y es muy importante en muchos procesos fisiológicos incluyendo la regulación de la eficiencia de fósforo ${ }^{9}$.

La fosfatasa ácida cataliza la hidrólisis de los sustratos fosforilados ${ }^{10}$ de acuerdo con la reacción

$$
\mathrm{RO}-\mathrm{PO}_{3}^{2-}+\mathrm{H}_{2} \mathrm{O} \longrightarrow \mathrm{ROH}+\mathrm{HPO}_{4}^{2-}
$$

Esta enzima cataliza la hidrólisis de los fosfomonoésteres, como el pnitrofenilfosfato, a través de un proceso cinético uni-biordenado, caracterizado por liberarse en primer lugar el $p$ nitrofenol y posteriormente el fosfato inorgánico. En esta secuencia de reacción se forma un complejo intermedio covalente enzima-fosfato, que es la etapa limitante de la reacción total del proceso catalítico ${ }^{11}$. El procesamiento térmico es uno de los métodos más comunes para lograr alimentos inocuos con una vida útil más extensa y su objetivo es destruir microorganismos patógenos y enzimas.
El conocimiento de la cinética de inactivación de las enzimas se puede utilizar para determinar su potencial como indicador intrínseco de temperatura-tiempo para lograr la inactivación microbiana ${ }^{12}$.La inactivación a diferentes temperaturas se realiza con el fin de obtener más información sobre la termo estabilidad de la enzima ${ }^{13,14}$, estos investigadores sugieren una equivalencia térmica como criterio para la evaluación de la influencia de diversos factores en la estabilidad de la enzima. La temperatura afecta no solo a la velocidad de la catálisis, sino también a la estabilidad del enzima, el equilibrio de todas las reacciones de asociacióndisociación y a las reacciones enzimáticas reversibles ${ }^{15}$. Para la investigación de los procesos de disociación-asociación y el mecanismo de termo inactivación se utilizan métodos cinéticos ${ }^{16-19}$. Se ha demostrado que la fosfatasa acida proveniente de diferentes fuentes como semillas de girasol, germen de trigo y semillas de maíz, es un dímero 20-22. La cinética de termo inactivación de las enzimas diméricas corresponde al mecanismo disociativo-asociativo de la enzima y la posterior disociación en subunidades inactivas ${ }^{23}$. conocimiento del mecanismo de termo inactivación permite proponer un mecanismo de estabilización de la Enzima 24 .

El objetivo de la presente investigación es estudiar la termoinactivación de la fosfatasa acida y mostrar cómo se 

experimento $\left(50^{\circ} \mathrm{C}, 55^{\circ} \mathrm{C}, 60^{\circ} \mathrm{C}\right.$ y $65^{\circ}$

realizan los cálculos de las diferentes constantes cinéticas involucradas en la termoinactivación de una enzima dimérica mediante el mecanismo asociativo-disociativo.

\section{MATERIALES Y MÉTODOS}

Reactivos. Se utilizó fosfatasa acida del germen de trigo de la firma Sigma con una actividad específica $>0.4 u n t / m g, p-$ nitrofenilfosfato de sodio hexahidratado de la firma Alfa Aesar A Jhonson Mathey Company, solución tampón acetato $\mathrm{pH} 5.5$, solución de hidróxido de sodio $0.1 \mathrm{M}$ reactivos analíticos marca Merck.

Determinación de la actividad de la fosfatasa acida. La dependencia de la actividad de la fosfatasa acida en función del $\mathrm{pH}$, se llevó a cabo para concentraciones de enzima $[E]=$ $1.38 \times 10^{-6} \mathrm{M}$ y sustrato $[\mathrm{S}]=1.67 \times 10^{-3} \mathrm{M}$, en un medio de $\mathrm{pH}$ variado entre (3.5; $4.5 ; 5.5 ; 6.5$ y 7.5 ) en buffer acetato, en el cual se disolvió las cantidades de enzima y sustrato determinados para cada concentración y por separado para cada $\mathrm{pH}$; luego en un tubo de ensayo debidamente rotulado se colocó $1 \mathrm{ml}$ de sustrato, se inició la reacción agregando $1 \mathrm{ml}$ de la enzima, después de un tiempo igual para cada reacción en los diferentes $\mathrm{pH}$, se detiene esta con $2 \mathrm{ml}$ de hidróxido de sodio y en un espectro fotómetro Génesis10 se hizo la lectura de la absorbancia a 405nm.

Termo inactivación. Se realiza con las concentraciones de enzima y sustrato anteriormente mencionadas. En un termostato, a la temperatura del
C) y en solución tampón acetato $0.1 \mathrm{M}$ $\mathrm{pH}$ 5,5 se colocaron en tubos de ensayo las concentraciones de sustrato. Experimentalmente los puntos iniciales de la curva cinética de termoinactivación se tomaron así: inicialmente el tubo de ensayo con 1 $\mathrm{mL}$ de sustrato se coloca en el termostato por dos minutos y luego se agregó la enzima, el tiempo de contacto entre el sustrato y la enzima inicialmente es de dos minutos, al cabo de los cuales se detiene la reacción con dos $\mathrm{mL}$ de hidroxido de sodio 0.1 M. En el segundo y posteriores puntos de la curva cinética lo único que cambia es el tiempo de contacto después de agregada la enzima (cuatro, seis, ocho, diez, doce minutos y así sucesivamente), transcurrido este tiempo se detiene la reacción con dos $\mathrm{mL}$ de hidróxido de sodio $0.1 \mathrm{M}$, después de un tiempo de reposo se efectuó la lectura de absorbancia a $405 \mathrm{~nm}$ en un espectrofotómetro Genesis10 para medir la cantidad de p-nitrofenol formado en cada isoterma.

\section{Resultados y Discusión}

Según ${ }^{25}$, las fosfatasas provenientes de la mayoría de las plantas, poseen una máxima actividad a valores de $\mathrm{pH}$ de 5.0-5.5. En la Fig 1 se muestra la dependencia de la actividad de la fosfatasa ácida respecto del $\mathrm{pH}$ a una temperatura de $22^{\circ} \mathrm{C}$, se observa que el máximo valor de $\mathrm{pH}$ corresponde a un valor de 5.5, que concuerda con las investigaciones $\mathrm{de}^{26}$. El aumento de la temperatura no afecta el $\mathrm{pH}$ óptimo de la Fosfatasa Acida, como se observa 
en la Fig 2 en donde se muestra la actividad de la fosfatasa Acida a temperaturas de 22,30 y $37^{\circ} \mathrm{C}$ y diferentes valores de $\mathrm{pH}$.

Al estudiar la actividad específica de la fosfatasa acida en función de su concentración, se observa que la actividad específica de la fosfatasa acida disminuye a medida que aumenta la concentración inicial de la enzima (Fig 3). Este hecho puede ser una consecuencia de la capacidad de la fosfatasa acida de formar asociados que poseen una menor actividad específica. Como la fosfatasa acida es un dímero activo que se disocia en dos monómeros inactivos, el estudio cinético a diferentes isotermas debe mostrar un punto de quiebre que correspondería a la formación de estos monómeros y por resolución de las ecuaciones diferenciales propias de este esquema; conocidas en los trabajos ${ }^{27}$, se determinan sus constantes cinéticas. En la Fig 4 se muestra la curva cinética de la termo inactivación de la Fosfatasa Acida a $55^{\circ} \mathrm{C}$.

Al representar esta curva en las coordenadas In $v \rightarrow(t)$, se observa un "punto de quiebre" (como lugar de intersección de las asíntotas lineales) cuando $t=\tau$ (aproximadamente a los 15 minutos) que demuestra que la inactivación de la fosfatasa acida incluye una etapa rápida y una etapa lenta, como se observa en la Fig 5. Dependencias análogas se obtuvieron para 50,60 y $65^{\circ} \mathrm{C}$

El punto de quiebre no depende de la concentración de la Enzima, la inclinación de los sectores iníciales de las curvas cinéticas para concentraciones de enzima entre $8.28 \times 10^{-7}$ y $5.52 \times 10^{-6} \mathrm{M}$ es el mismo, sin embargo la inclinación en el segundo sector es mayor entre menor sea la concentración de la enzima, como se observa en la Fig 6.

Esta forma de las curvas cinéticas es típica de la inactivación de enzimas por el mecanismo disociativo-asociativo ${ }^{28}$ y para una enzima dimérica el esquema cinético tiene la siguiente forma (esquema 1)

(1)

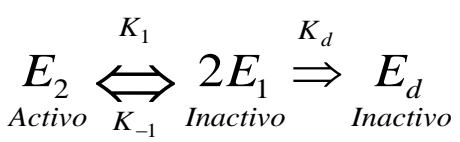

En donde: $E_{2}$-dímero activo, $E_{1-}$ monomero inactivo, capaz de formar un dímero activo y $E_{d}$ enzima denaturizada, $K_{\text {dis }}=\left(k_{1} / k_{-1}\right)$ constante de disociación de la fosfatasa acida; $k_{d}$ constante de velocidad de denaturación.

En concordancia con el mecanismo de la termo inactivación disociativa, la variación de la actividad de la enzima se puede comparar: 1.-Para $t<\tau$ con la disociación reversible de la forma dimérica activa en subunidades inactivas hasta establecerse el equilibrio en el punto de quiebre $\tau$ y 2 .para $t>\tau$ con el proceso de inactivación irreversible de los monómeros-denaturizados. La posición del "punto de quiebre" y la inclinación de las curvas cinéticas en las coordenadas de una ecuación de primer orden permiten determinar tres parámetros cinéticos del esquema 1 . Estos serán las constantes de 
22

velocidad $k_{1}, k_{-1}$ y $k_{\mathrm{d}} \mathrm{O}$ dos constantes de velocidad $k_{1}$ y $k_{d}$ y una constante de equilibrio $K_{\text {dis }}$

El cálculo de la constante de disociación $K$ dis y las constantes individuales del proceso descrito por el esquema (1) se realizan por las ecuaciones propuestas por ${ }^{28}$.

$$
K_{\text {dis }}=\left(\frac{K_{1}}{K_{-1}}\right)=4\left[E_{0}\right] \frac{\left(V_{0}-V_{\tau}\right)^{2}}{\left(V_{0}+V_{\tau}\right)}
$$

En donde: $\left[E_{o}\right]$ es la concentración inicial de la enzima dimérica; $v_{0}$ y $v_{\tau}$ las correspondientes velocidades para $t=0$ y $t=\tau$ (punto de quiebre en la curva cinética de la Fig 5).

La constante $k_{1}$, que caracteriza la constante de velocidad de disociación de los dímeros, se determina mediante la ecuación $(2)^{28}$

$$
2\left(\frac{V}{V_{\mathrm{O}}}\right)-\left(\frac{1}{2}\right)\left(\frac{V}{V_{\mathrm{O}}}\right)^{2}=\frac{3}{2}-{ }_{(2)} K_{1} t
$$

Aquí $v_{0}$ es la velocidad de la reacción enzimática para $\mathrm{t}=0$; $\mathrm{v}$ es la velocidad en cualquier tiempo $t<\tau$.

En la Fig 7 se muestra la determinación gráfica de $k_{1}$ según la ecuación (2) para $55^{\circ} \mathrm{C}$ y $60^{\circ} \mathrm{C}$. La determinación de la constante de denaturación $K_{d}$ se realiza por la ecuación (3)

$$
K_{d}=B \frac{\left(V_{0}+V_{\tau}\right)}{2\left(V_{0}-V_{\tau}\right)}
$$

Básicas.2015.13(1):16-26

En esta ecuación $B$ es la tangente de la inclinación en el punto de quiebre de las curvas cinéticas (fig 5)

Los valores de $k_{1}$ y demás parámetros cinéticos de la termo inactivación de la fosfatasa acida se presentan en la Tabla I.

Para demostrar la reversibilidad de la primera etapa del proceso disociativoasociativo, la enzima se sometió a una temperatura de $55{ }^{\circ} \mathrm{C}$ durante quince minutos (tiempo en el que se observa el punto de quiebre) e inmediatamente se colocó en frio (hielo) durante quince minutos al cabo de los cuales se determinó su actividad, encontrándose una recuperación del $90 \%$ de su actividad después de sometida la enzima a un calentamiento severo.

El conocimiento de las magnitudes $k_{1} y$ $k_{d}$ a diferentes temperaturas permite calcular las energías de activación correspondiente a las etapas del esquema 1

\section{Conclusiones:}

1.-Las curvas cinéticas en coordenadas semilogarítmicas de una ecuación de primer orden tienen un punto de quiebre.

2.- Se calculan los parámetros cinéticos característicos de la termo inactivación de una enzima dimérica.Los valores de $k_{1}$ correspondientes a la constante de velocidad de disociación de la fosfatasa acida que determinan la estructura del dímero, aumentan con la temperatura 
3.-La energía de activación de la disociación del dimero a monómero de la fosfatasa acida es de $23.22 \mathrm{KJ} / \mathrm{mol}$.

\section{AGRADECIMIENTOS}

Los autores agradecen a la Rectoría de la Universidad de Pamplona por su apoyo económico en la realización de esta investigación y al laboratorio de Biomoléculas de la Universidad de Pamplona.

\section{Referencias bibliográficas}

1.-Aoyama $\mathrm{H}$, Martins Cavagisa AD, Taga EM, Ferreira CV. Endogenous lectin as a possible regulator of the hydrolysis of physiological substrates by soybean seed acid phosphatase. Phytochemistry.2001;58 (2): 221-225.

2.-Dos Prazeres JN, Ferreira CF, Aoyama $\mathrm{H}$. Acid phosphatase activity during germination of Glycine max seeds. Plant. Physiol. Biochem. 2004 ;42(1): 15-20.

3.-Guimaraes LHS, Terenzi HF, Joao J, Leone FA, Polizeli ML. Characterization and properties of acid phosphatase with phytase activity produced by Aspergillus caespitosus. Biotechnol. Appl. Biochem.2004; 40 (2): 201-207.

4.-Kaur P, Lingner A, Singh B, Böer E, Polajeva J, Steinborn G, Bode R, Gellissen G., Satyanarayana T, Kunze G.APHO1 from the yeast Arxula adeninivorans encodes an acid phosphatase of broad substrate specificity. Antonie van Leeuwenhoek. 2007;91(1):45-55.

5.-El Hadef el Okki S; Philippon C; Mouthon G.Optimization of the determination of muscle acid phosphatase in the postmortem chicken. Study of the stability of enzyme activity during meat storage at $4{ }^{\circ} \mathrm{C}$ and $-20^{\circ} \mathrm{C}$. Ann. Rech. Vet.1987. 18 (1):
S Básicas.2015.13(1):16-26

6.-Incze K, Kormendy L, Kormendy I, Zsanoczay G.Consideration of critical microorganisms and indicator enzymes in connection with the pasteurization of meat products. Meat Science.1999; 51(2): 115121.

7.-Jung S, Ghoul M, De Lamballerie-Anton $M$. Changes in lysosomal enzyme activities and shear values of high pressure treated meat during ageing. Meat Science.2000;56(3):239-246.

8.-Kuda T, Tsuda N, Yano T.Thermal inactivation characteristics of acid and alkaline phosphatase in fish and shellfish. Food Chemistry.2004; 88(4): 543-548.

9.-Yan X, Liao H, Trull MC, Beebe SE, Lynch JP. Induction of a major leaf acid phosphatase does not confer adaptation to low phosphorus availability in common bean. Plant Physiol. 2001;125(1):19011911.

10.-Mcgeary RP, Vella PM, Jeffery YW, Guddat LW, Gerhard S. nhibition of purple acid phosphatase with alphaalkoxynaphthylmethylphosphonic acids. Bioorganic \& Medicinal Chemistry Letters. 2009;19 (1):163-166.

11.-Guija E, Arauco F, Hielke HM, Soberón M. Efecto del glicerol sobre la catálisis por fosfatasa ácida de bajo peso molecular de hígado de alpaca (Lama pacos). An Fac Med Lima.2007; 68(4): 307-313.

12.-Sissel OJ, Dagbjorn S, Torstein S, Hendrickx M E.Thermal Inactivation kinetics of acid phosphatase (ACP) in cod (Gadus morhua). European Food Research and Technology.2007; 224(3), 315-320.

13.-Greco G, Pirozzi D, Gianfreda L. . Thermal equivalence criteria in the chemical deactivation and stabilization of 
acid phosphatase. Enzyme Microb. Technol.. 1991;13(4):353-358.

14.-Vrabel P, Polakovic M, Tefuca V, Bale V.Analysis of mechanism and kinetics of thermal inactivation of enzymes:Evaluation of multitemperature data applied to inactivation of yeast invertase.Enzyme and Microbial Technology.1997;20(5): 348-354.

15.-Fenema OR. 2000.Food Chemistry. Third edition, Ed Marcel Dekker,Inc. New York, 551.

16.-Siksnis V, Mozhaev VV, Galkantaite N, Melik-Nubarov NS, Denis G, Martinek K.. Unusual (zig-zag) temperature dependence of the rate constant for irreversible thermoinactivation of hydrophilized enzymes. Collect. Czech. Chem. Commun. 1990;55(5):1366-1371.

17.-Owusu RK \& Berthalon N. A test for the two-stage thermoinactivation model for chymotrypsin. Food Chem.1993;48(3): 231235.

18.-Nohara D, Yamada T, Watanabe A, Sakai T.Equilibrium and kinetic studies on reversible and irreversible denaturation of micrococcal nuclease. Biotechnol. Bioeng.1994; 44 (3): 276-82.

19.-Mozhaev VV, Melik-Nubarov NS, Levitsky VY, Siknis VA, Martinek K.High stability to irreversible inactivation at elevated temperatures of enzymes covalently modified by hydrophilic reagents: a-chymotrypsin. Biotechnol Bioeng. 1992; 40 (6): 650-662.

20.-Waymack PP \& Van Etten RL. (1991). Isolation and characterization of a Homogeneous isoenzyme of wheat germ acid phosphatase. Arch Biochem Biophys. $1991 ; 288(2): 621-633$.
Fialho $\mathrm{E}$. Induction of acid phosphatase activity during germination of maize (Zea mays) sedes. Plant Physiology and Biochemistry.2006; 44: (7-9), 467-473.

22.-Tanemura $Y$ \& Yoshino M. Regulatory role of polyamine in the acid phosphatase from potato tubers. Plant Physiol Biochem. 2006;44 (1): 43-8.

23.-Torshin IYu, Poltorak OM, Chukhrai ES. Estabilización de la Fosfatasa Alcalina por iones de Magnesio. Vestnik. Mosk. Univ. Serie 2. Química.1996; 37(5): 335340

24.-Lundovskikh IA, Dementieva El, Ugarova NN. кинетика и механизм термоинактивации рекомбинантной люциферазы светляков. Luciola. Mingrelic. Vestnik. Mosk. Univ. Serie 2. Quimica.2006;41 (6): 362-366.

25.-Jagiello I, Donella-Deana A, Szczegielniak J, Pinna L, Muszynska G. Identification of protein phosphatase activities in maize seedlings. Biochim et Biophys.1992;1134(2): 129-136.

26.-Tso SC, Chen YR. Isolation and characterization of a group III isozyme of Acid Phosphatase from rice plants. Bot. Bull. Acad. Sin. 1997;38: 245-250.

27.-Poltorak OM \& Chukhrai ES. 2002. Curso especial de cinética enzimática, editorial Moskoskovo Gosudarstvennaia Universitieta "M.V.Lomonosov". Moscow.

28.-Poltorak OM, Chukhrai ES, Torshin IYu, Nakkar S, Veselova M.Chemistry stabilization of alkaline phosphatase by magnesium ions.Moscow University chemistry bulletin..1998;53(4):18-21

\section{1.-Senna R, Simonin V, Silva-Neto MAC,}


Fig 1. Dependencia de la actividad de la fosfatasa acida en función del pH

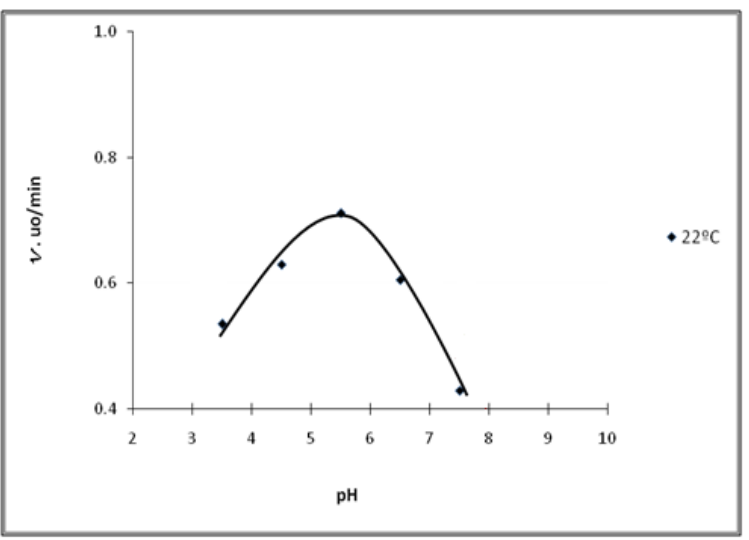

Fig 2.Dependencia de la temperatura en la actividad de la fosfatasa acida en función del $\mathrm{PH}$

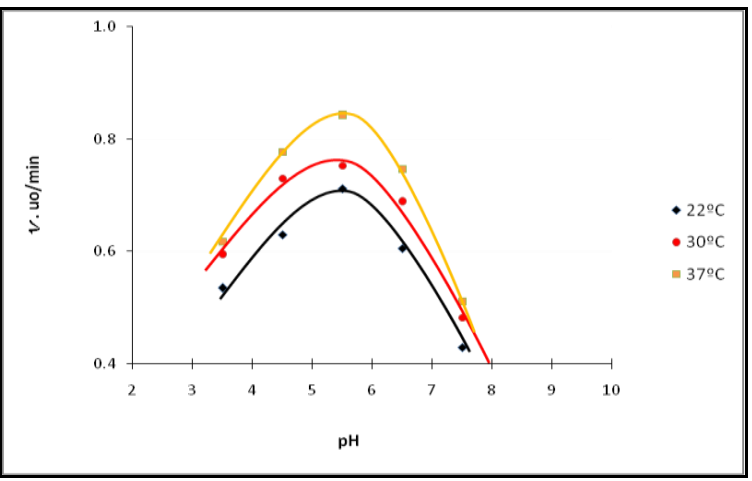

Fig 3 Actividad específica de la fosfatasa acida en función de la concentración inicial de la enzima para un $\mathrm{pH}$ de 5.5

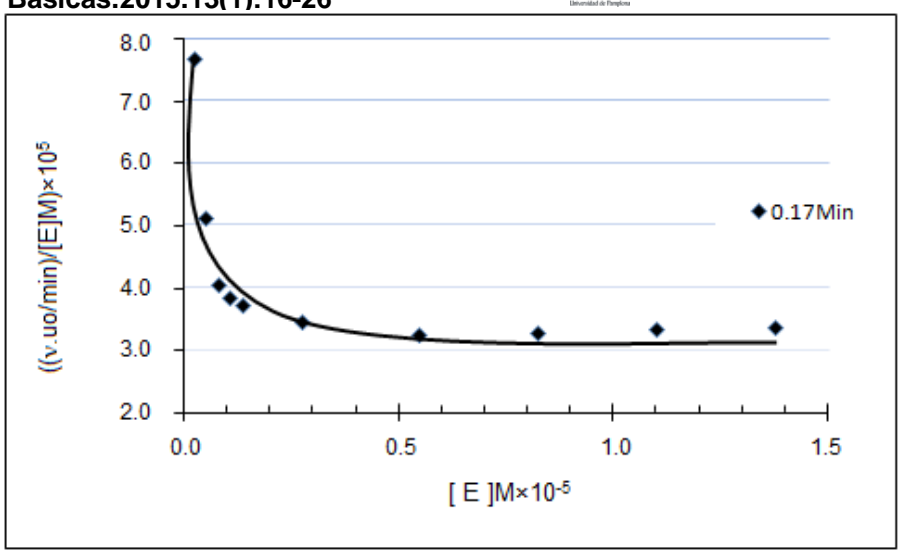

Fig 4. Curva cinética de la termoinactivación de la fosfatasa acida en un buffer acetato $0.1 \mathrm{M} \mathrm{a}$ un $\mathrm{pH}$ de $5.5 \mathrm{y}$ temperatura de $55^{\circ} \mathrm{C}$

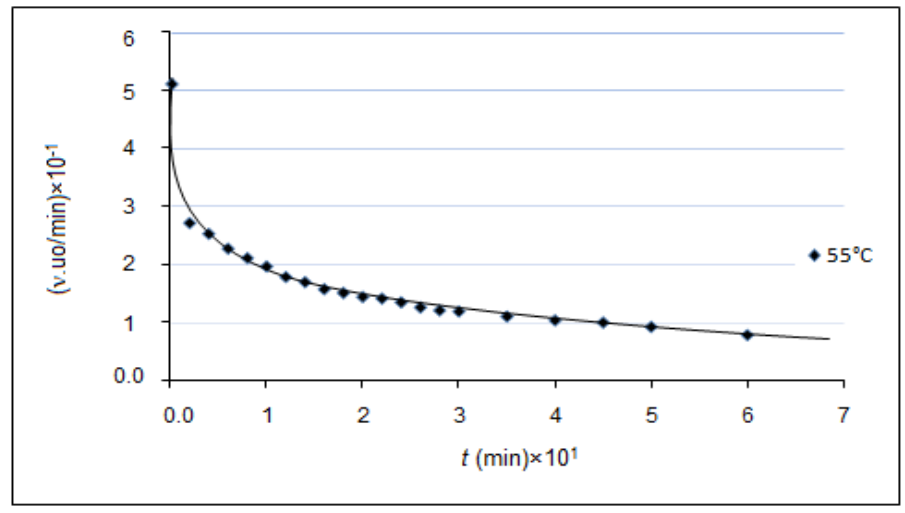

Fig 5. Curva cinética de la termoinactivación de la fosfatasa acida en coordenadas semilogaritmicas a $55^{\circ} \mathrm{C}$ y $\mathrm{pH} 5.5$

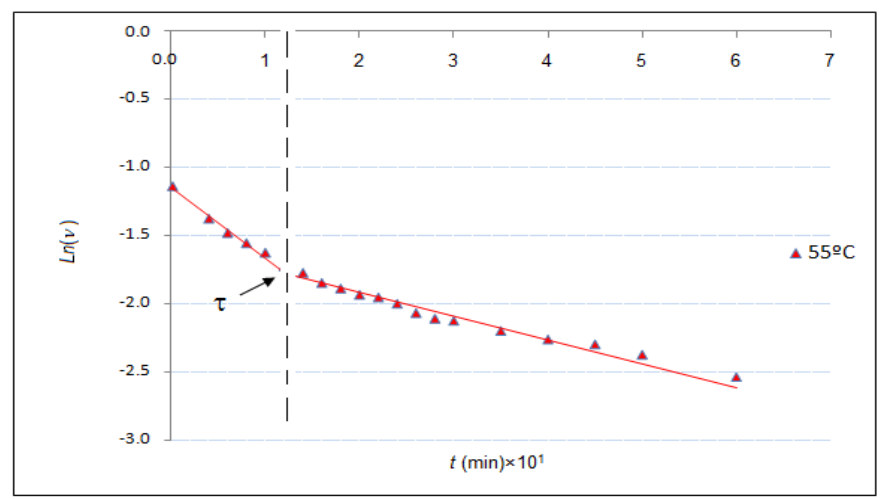


Fig 6. Curvas cinéticas de la termoinactivación de la fosfatasa acida en coordenadas semilogaritmicas, a $55^{\circ} \mathrm{C}$ y $\mathrm{pH} 5.5$ para diferentes concentraciones de enzima

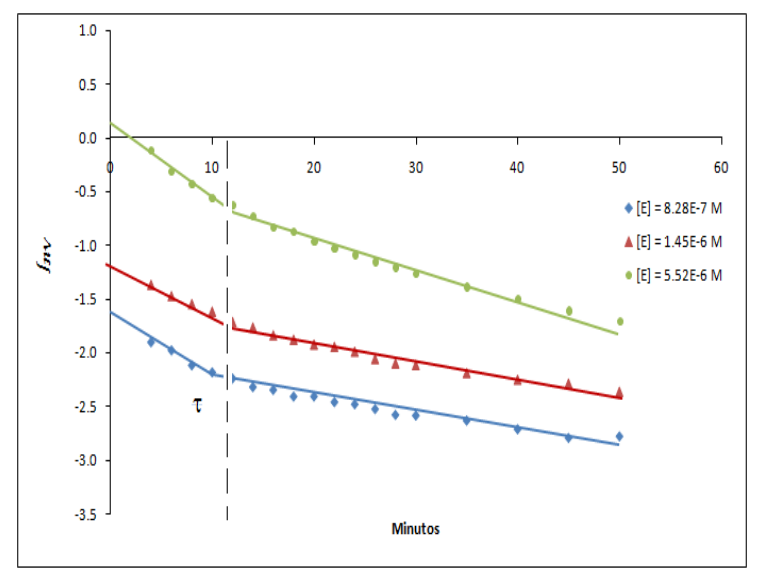

Fig 7.-Determinación gráfica de $K_{1}$ según la ecuación (2) para $50^{\circ} \mathrm{C}$ y $65^{\circ} \mathrm{C}$

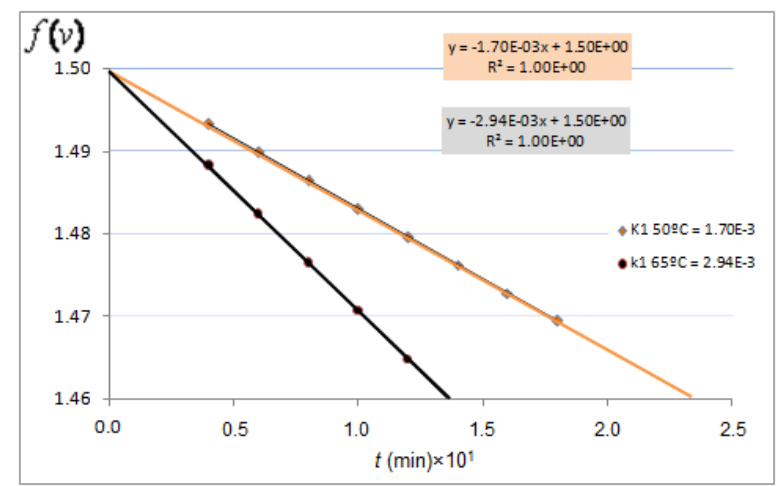

Tabla I. Parámetros cinéticos de la inactivación de la fosfatasa acida a diferentes temperaturas

\begin{tabular}{|l|l|l|l|l|}
\hline${ }^{\circ} \mathrm{C}$ & $K_{1}=10^{-5} \mathrm{~s}^{-1}$ & $K_{\mathrm{d}}=10^{-4} \mathrm{~s}^{-1}$ & $K_{-1}=10^{-3} \mathrm{~s}^{-1}$ & $K_{\text {dis }}=10^{-9} \mathrm{~s}^{-1}$ \\
\hline 50 & 2.83 & 4.55 & 7.18 & 3.94 \\
\hline 55 & 3.02 & 4.67 & 8.04 & 3.75 \\
\hline 60 & 3.52 & 3.62 & 5.41 & 3.51 \\
\hline 65 & 4.90 & 3.23 & 5.12 & 9.57 \\
\hline
\end{tabular}

Quijano Parra, Alfonso: Ph.D

Profesor Asociado. Investigador Asociado .Universidad de Pamplona, PamplonaColombia, Facultad de Ciencias Básicas. Laboratorio de Control de Calidad. Grupo de Investigación en Química. alfonsoquijanoparra@unipamplona.edu.co

Martínez Chavez, Oscar Luis. Ms C Universidad de Pamplona, PamplonaColombia, Facultad de Ciencias Básicas. Grupo de Investigación en Química.

Arbeláez Ramirez,Luis Fernando.Ph.D Profesor Titular. Investigador Asociado. Universidad de Pamplona, PamplonaColombia, Facultad de Salud. Grupo de Investigación en Química. Laboratorio de Biomoléculas. 